\title{
CARGA IMEDIATA EM PRÓTESE TOTAL IMPLANTOSUPORTADA: REVISÃO DE LITERATURA.
}

\author{
Sueli de Souza Monteiro França ${ }^{1}$, Eber Coelho Paraguassu ${ }^{2 *}$
}

\section{$\underline{\text { ARTIGO DE REVISÃO }}$}

Resumo

Por muito tempo instalava-se somente implantes dentário de forma submersa durante o período de cicatrização. Com o passar dos anos, novos estudos foram realizados e comprovados a eficiência implantar de implante com carga imediata funcional, diminuindo assim o período entre a fase cirúrgica e protética. O uso da técnica de carga imediata precisa ser bem planejado de acordo com as especificidades de cada paciente, visto que, cada caso possui suas particularidades fisiológicas, histológicas e anatômicas. A carga imediata é um procedimento que altera o protocolo original de Branemark, que recomenda um período de reparo tecidual de três a seis meses. No princípio alguns autores consideravam que a colocação de carga imediata nos implantes comprometeria a osseointegração, mas depois se observou que a cicatrização submersa não é essencial para que ela ocorra e sim um controle dos micros movimentos na interface tecido ósseo-implante, o qual é considerado um fator crítico para seu sucesso. Para viabilizar o protocolo de carga imediata sobre implantes, a prótese deverá ser instalada nas primeiras 24 a 48 horas ou até duas semanas após a cirurgia. Nas literaturas atuais demonstram sucesso de carga imediata em prótese total implantosuportada, sendo necessário alguns requisitos a serem seguidos, como: estabilidade primária de $40 \mathrm{~N}$ de torque no mínimo, quantidade e qualidade óssea disponível, ausência de cantiléver, condição sistêmica do paciente, são algumas situações necessárias para que o implante seja osseointegrado e adquire o sucesso clínico. O objetivo deste artigo é realizar uma revisão de literatura sobre carga imediata em prótese total implantosuportada.

PALAVRAS-CHAVE: Carga imediata; estabilidade primária; osseointegração. 


\title{
IMMEDIATE LOAD IN TOTAL IMPLANT PROSTHESIS SUPPORTED: LITERATURE REVIEW
}

\begin{abstract}
For a long time, only submerged dental implants were installed during the healing period. Over the years, new studies were carried out and proved the efficiency of implanting an implant with immediate functional load, thus reducing the period between the surgical and prosthetic phases. The use of the immediate loading technique needs to be well planned according to the specificities of each patient, as each case has its physiological, histological and anatomical particularities. Immediate loading is a procedure that changes the original Branemark protocol, which recommended a tissue repair period of three to six months. At first, some authors considered that placing immediate load on the implants would compromise osseointegration, but later it was observed that submerged healing is not essential for it to occur, but a control of micro movements at the bone tissue-implant interface, which is considered a critical factor in your success. To enable the immediate loading protocol on implants, the prosthesis must be installed within the first 24 to 48 hours or up to two weeks after surgery. Current literature demonstrates immediate loading success in implant-supported complete dentures, requiring some requirements to be followed, such as: primary stability of at least $40 \mathrm{~N}$ torque, available bone quantity and quality, absence of cantilever, systemic condition of the patient, are some situations necessary for the implant to be osseointegrated and achieve clinical success. The aim of this article is to perform a literature review on immediate loading in implant-supported complete dentures.
\end{abstract}

Keywords: immediate charge; primary stability; osseointegration.

Instituição afiliada: ${ }^{1}$ Pós-graduando do Curso de Especialização em Implantodontia da Universidade Uniavanrepresentado por GOE /AP. ${ }^{2}$ Coordenador da pós-graduação em Implantodontia da Universidade Uniavan, representado por GOE

/AP.

Dados da publicação: Artigo recebido em 12 de Outubro, revisado em 17 de Novembro, aceito para publicação em 03 de Dezembro e publicado em 31 de Janeiro de 2022

DOI: https://doi.org/10.36557/2674-8169.2022v4n1p14-34

Autor correspondente: Éber Coelho Paraguassu paraguassutans@gmail.com

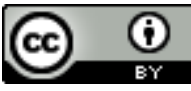

This work is licensed under a Creative Commons Attribution 4.0 International

License. 


\section{INTRODUÇÃO}

Atualmente pacientes edêntulos total têm procurado com mais frequência a reabilitação protética sobre implante. Em busca de autoestima, estética e funcional. Ecom o surgimento de protocolos de carga imediata em prótese total implantosuportada, há possibilidade de uma reabilitação oral em um curto espaço detempo em relação ao protocolo convencional de implantodontia.

O surgimento dos implantes com carga imediata ocorreu visando proporcionar ao paciente mais conforto. Neste, logo no ato cirúrgico é possível que se instale o implante e a prótese $^{1}$.

Inicialmente, alguns autores acreditaram que esse método prejudicaria a cicatrização e a remodelação óssea do trauma cirúrgico. Devido a manutenção do contato direto do osso sem interposição de fibras com a face exterior do implante emcargas imediatas, mudou-se a visão relacionada aos implantes de dois momentos ${ }^{1}$.

Altas taxas de sobrevivência de implantes com carga imediata foram inicialmente descritas por volta de 1980 quando o protocolo de uma fase cirúrgica ganhou popularidade 2 .

Nas últimas décadas, vários autores confirmam a possibilidade de osteointegrar implantes em forma de raiz dentária, mesmo se emergirem do osso através do tecidomole durante a remodelação óssea inicial. Desta forma evita a segunda fase cirúrgica, e a fase protética é antecipada, oferecendo bem estar psicológico e funcional para o paciente. Esta abordagem cirúrgica tem sido denominada de carga Imediata. Grandes evidências científicas têm demostrado que pode ser atingido o sucesso com esse protocolo ${ }^{3,4,5,6,7,8}$.

Existe esta alternativa de tratamento implantar de forma imediata funcional, porém, há restrições quanto a saúde sistémica do paciente, a quantidade e qualidadeóssea do leito cirúrgico, a estabilidade do implante, ausência de cantiléver são algumas situações necessárias para que o implante seja osseointegrado e adquire o sucesso clínico.

O presente trabalho se justifica por demonstrar através de revisão de literatura os requisitos que um paciente precisa ter para receber um implante dentário com ativação de carga imediata. 
O objetivo deste artigo é o estudo de revisão de literatura sobre o tema carga imediata em prótese total implantosuportada em mandíbula e maxila, bem como discorrer sobre as condições para o seu uso.

\section{MÉTODOS}

Foi realizada uma varredura nos bancos de dados Google Acadêmico, Scielo, Pubmed e Bireme, utilizando as seguintes palavras chaves: prótese protocolo carga imediata e implante. O público-alvo sendo o edentulismo mandibular ou maxilar.

\section{REVISÃO DE LITERATURA}

\section{DEFINIÇÃO DE CARGA IMEDIATA}

A carga imediata com próteses fixas de arco completo se torna benéfica para os pacientes, já que permite reduzir os tempos de espera até a reabilitação protética,sempre que não comprometa o sucesso dos implantes ${ }^{9}$.

Estética imediatas a não necessidade utilizar uma prótese provisória removível não precisa de segunda cirurgia ${ }^{10}$.

Carga imediata em Implantodontia pode ser definida como sendo a instalação de próteses sobre implantes, imediatamente após cirurgia, sem que tenha ocorrido a osseointegração. O primeiro trabalho publicado sobre a carga imediata em implantesconcluiu que a ativação precoce não afeta o prognóstico do tratamento, sendo os implantes mandibulares com maior possibilidade de falha os colocados o mais distais, suportando cantilever ${ }^{11}$.

Existem alguns aspectos fundamentais para o sucesso deste tratamento que são: implantes com comprimento mínimo de $10 \mathrm{~mm}$, a importância da utilização de uma estrutura metálica rígida para uma melhor estabilidade e resistência da prótese provisória, sendo que os cantilevers devem ser evitados, e as próteses provisórias devem, sempre que possível, ser do tipo parafusada. Não se deve remover a prótesesobre implante submetido à carga imediata, precocemente, ou seja, esperar um período mínimo de 4 a 6 meses, evitando macro e micromovimentos nos implantes, oque acarretaria interferências na interface osso-implante e consequentes falhas ${ }^{12}$.

A perda ou ausência dos dentes afeta o indivíduo desde a perspectiva física, social e psicológica, especialmente no âmbito da autoestima, portanto, a reconstrução 
dentaria de pacientes total ou parcialmente desdentados, é de grande importância desde a perspectiva biopsicossocial de saúde ${ }^{13}$

Para viabilizar o protocolo de carga imediata sobre implantes, a prótese deverá ser instalada nas primeiras 24 a 48 horas ou até duas semanas após a cirurgia ${ }^{14,16}$.

Reduz o tempo de tratamento, não é necessário o desconforto do uso de próteses removíveis provisórias e procedimentos de reembasamento. A estabilidade primária entre implante e o osso, se manterá pela estabilidade secundária obtida por meio da utilização da prótese fixa. A principal causa da formação de um encapsulado fibroso ao invés da osseointegração são os micros e macromovimentos do implante em relação ao osso circundante. Isto leva a perda dos implantes tanto no protocolo dedois estágios, como no de um estágio cirúrgico. Micromovimentos de 10 a 28 micronspoderiam prevenir esse processo, com a carga sendo direcionada de forma axial ou transversa em relação ao implante ${ }^{14}$.

A implantação deverá ser executada em osso com espessura e qualidade adequadas, ou seja, osso tipo I, II e III. Igualmente, um contato maior e mais rápido pode ser atingido com implantes que incorporam tratamento de superfície do que implantes de superfície lisa. O ideal seria implantar cinco implantes na mandíbula, com comprimentos de pelo menos $8,5 \mathrm{~mm}$ (plataforma larga) ou $10 \mathrm{~mm}$ (plataforma regular), sendo que deveriam receber um torque mínimo de $40 \mathrm{Ncm}^{2,14}$.

A qualidade óssea influencia no processo de osseointegração. Classificam a qualidade óssea em 4 tipos, de acordo com a estrutura morfológica: Tipo I, compostode osso cortical muito espesso; Tipo II, camada espessa de osso cortical que envolve um núcleo de osso trabecular denso; Tipo III, camada fina de osso cortical que envolveum núcleo de osso trabecular de boa resistência; e Tipo IV, camada muito fina de ossocortical com osso trabecular de baixa densidade de baixa resistência ${ }^{15}$.

Figura 1. Classificação dos quatro tipos ósseos de acordo com estrutura morfológicae características celulares do tecido ósseo.

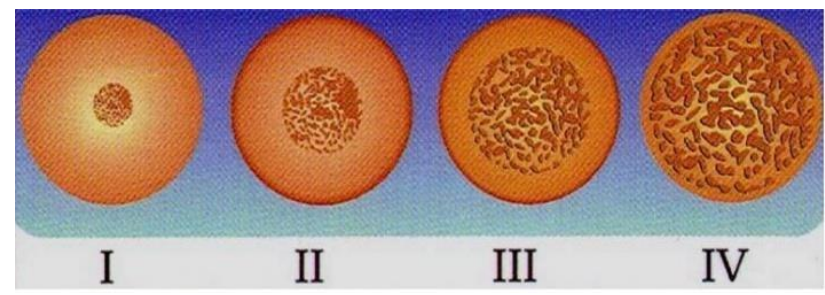

Fonte: Lekholm \& Zarb (1985). 


\section{CONCEITO DE OSTEOINTEGRAÇÃO}

Foi a partir de uma experiência com um coelho que o professor Per-Ingvar Branemark descobriu que o metal titânio era biocompatível com o osso. A investigaçãocomeçou em 1965, quando Branemark, chefiando um grupo de pesquisadores da Universidade de Gotemburgo, na Suécia, buscou desenvolver novos procedimentos cirúrgicos para deficiências físicas em seres humanos, dando início os estudos que culminaram com a descoberta da osseointegração 17 .

Quando um implante é colocado no osso, existe um contato direto, ou seja, estão osteointegrados. Inclusivamente, se a instrumentação for feita de forma a que aosteotomia tenha um diâmetro ligeiramente menor que o implante, de maneira a que este tenha um "press-fit" contra o tecido ósseo, isto representa um ótimo contato implante-osso em períodos de cicatrização iniciais. Este fenómeno foi descrito como contato ósseo primário. A análise histológica revela um contato intímo do osso com a superfície do implante, incluindo deformação lamelar plástica, sistema de Harvers alongados e microfracturas no osso ${ }^{18}$.

Ao investigar a microcirculação sanguínea em tíbias de coelho com ajuda de uma câmara de observação em titânio, Branemark percebeu que o metal e o osso seintegravam perfeitamente, sem que houvesse indícios de rejeição. A partir desta observação, o pesquisador passou a desenvolver cilindros personalizados para seremimplantados na tíbia de coelhos e cães 19 .

Uma grande variedade em tamanhos e formas de implantes tem evoluído e melhorado os resultados clínicos. A pesquisa contínua revela que alterações sutis na forma, no comprimento e na largura dos implantes poderiam influenciar o sucesso daossseointegração ${ }^{20}$

\section{DEFINIÇÃO DE ESTABILIDADE PRIMÁRIA}

A estabilidade primária pode ser definida como o travamento mecânico do implante no momento de sua instalação, o qual é relacionado com a qualidade e quantidade óssea local, tipo do implante e técnica de colocação usada. Uma baixa estabilidade primária resulta em micro movimentações do implante no osso, empobrece o processo de cicatrização e impede que a osseointegração ocorra. Diante disso, percebe-se que uma estabilidade primária insuficiente predispõe uma 
estabilidade secundária escassa ou até mesmo nula, levando à falha na osseointegração 21.

A estabilidade primária é necessária para evitar a formação de fibras ao redor do implante, que ocorre devido ao excesso de micromovimento no período deosseintegração, havendo um limite tolerado de micromovimentos, que varia entre $50 \mu \mathrm{m}$ e $150 \mu \mathrm{m}$. É necessário que os implantes tenham um contato suficiente com o osso, para que o total de cargas transferidas do implante ao mesmo mantenha-se abaixo do limite de microdanos. 0 limiar de micromovimentação de $100 \mu \mathrm{m}$ para os implantes de superfície lisa deve ser respeitado e para os implantes de superfície tratada, é tolerável variar entre 50 e $150 \mu \mathrm{m}^{22}$.

\section{A SUPERFÍCIE DO IMPLANTE}

As rugosidades dos implantes possibilitam uma maior ligação e entrelaçamento das fibrinas e origina caminhos para a migração das células adjacentes em direção àsuperfície do implante. Os tratamentos das superfícies visão alterar a morfologia superficial, atrair e permitir as ligações com as células préosteogênicas, entre elas a fibronectina. Com o aprisionamento desta na superfície do implante, esta célula servede ponte de atração e ligação com as células préosteoblásticas. Procura-se obter propriedades superficiais adequadas para se ter maiores concentrações possíveis deproteínas. O controle das características da superfície dos implantes é importante para garantir adequada adsorção de proteínas, adesão, espalhamento, crescimento e ativação celular ${ }^{23}$.

A topografia da superfície dos implantes diz respeito ao grau de rugosidade e à orientação das irregularidades da superfície. Os implantes originais de Brånemark eram usinados com uma mínima rugosidade na superfície, entre $0,5 \mu \mathrm{m}$ e 1,0 $\mu \mathrm{m}$. Por muito tempo, este implante foi reconhecido como o padrão ouro, baseado em muitose bons estudos clínicos longitudinais 24,25 .

Vários são os artigos que concordam que implantes com superfícies rugosas tanto no corpo como no colo e uma profunda micromorfologia da superfície são superiores em manter a estabilidade primária do que superfícies maquinadas, o que os torna primeira opção em osso de má qualidade e protocolos de carga imediata ${ }^{26,}{ }^{27}$. 
Os primeiros implantes, não apresentavam tratamento e modificações na sua superfície. Eram chamados de Usinados ou Maquinados e possuíam apenas ranhuras decorrentes do processo de fabricação ${ }^{28}$.

\section{DESENHO E TAMANHO DO IMPLANTE}

Ao avaliarem a importância do desenho do implante na osseointegração, demonstraram que os implantes rosqueados apresentam maior área de superfície, oferecendo assim maior porcentagem de contato osso-implante e melhor distribuição de forças, promovendo maior estabilidade primária nos implantes e diminuindo a micromovimentação, fatores que são fundamentais para a utilização da carga imediata 29.

O comprimento do implante é especialmente relevante em osso pouco denso. $\mathrm{O}$ benefício de aumentar o comprimento não está em aumentar a interface osso- implante na zona crestal, mas sim em aumentar a estabilidade inicial do implante ${ }^{30}$.

No estudo de Degidi et al, os autores concluiram que a carga imediata em implantes de tamanho standard $(13 \mathrm{~mm})$ têm altas taxas de sobrevivência, mas são estatisticamente piores do que carga imediata em implantes mais longos. Concluiram ainda que implantes de comprimento standard ou longos mostram resultados de confiança em carga imediata em osso de má qualidade, apesar de maior reabsorçãoóssea ser esperada ${ }^{31}$.

As roscas foram incorporadas nos implantes para melhorar a estabilidade inicial, aumentar a área de superfície e distribuir o stress favoravelmente. Apesar da evidência científica não ser clara sobre o efeito da forma das roscas na estabilidade inicial do implante, sabe-se que influenciam a área de superfície funcional, especialmente importante no caso de osso de menor densidade. Três formas básicasde roscas incluem: (A) rosca em " $V$ ", (B) rosca em trapézio inverso, (C) rosca quadrada 32.

Figura 2: Vidyasagar, L. e Apse, P., 2004

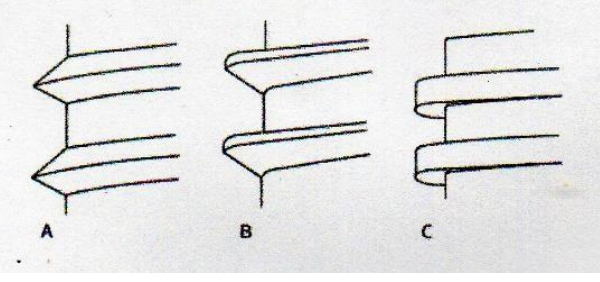




\section{INDICAÇÕES DE CARGA IMEDIATA}

\section{PACIENTES DESDENTADOS TOTAIS E PACIENTES DESDENTADOS PARCIAIS}

\section{Mandíbula}

Ostman, P., em 2000, relatou que a carga imediata em reabilitações fixas na mandíbula tinha taxas de sucesso entre $95 \%$ e $100 \%{ }^{33}$.

Em 2004, Cochran e os seus colaboradores definiram no consensus, que a carga imediata de uma sobredentadura e reabilitação fixa mandibular sobre 4 implantes na área intermentoniana com uma barra de fixação rígida, eram procedimentos previsíveis e bem documentados ${ }^{34}$.

\section{Maxila}

Maxilas totalmente desdentadas têm muito menos estudos publicado sobre carga imediata, comparativamente à mandíbula ${ }^{33}$, sendo que vários são os autores que referem não existirem dados suficientes que suportem a carga precoce ou imediata com sobredentaduras. Consideram, portanto, este procedimento como um procedimento experimental ${ }^{34,35,36}$

Em 2005, é demostrado a pouca literatura existente sugeria taxas de sucesso limitadas para carga imediata na maxila ${ }^{37}$.

Em 2005, concluíram que a maxila edêntula era um local apropriado para cargaimediata, com reabilitações fixas totais do arco sobre, no mínimo, 6 implantes ${ }^{2}$. Em 2008 sugeria no mínimo 8 implantes ferulizados para reabilitações fixas de carga imediata na maxila ${ }^{38}$.

\section{VANTAGENS DA CARGA IMEDIATA}

As vantagens da carga imediata são segundo vários autores 39,40,41,42,43,44:

- Significativa redução do tempo total de tratamento;

- $\quad$ Elimina a necessidade do uso prótese removível transitória, reduzindo do risco detrauma ou exposição dos implantes submersos pela prótese;

- Redução do trauma para o paciente (morbilidade) com a eliminação de consulta deexposição do implante;

- Benefícios psicológicos para o paciente que não passa pelo stress emocional nemdesconforto funcional de estar edêntulo ou com uma prótese removível; 
- Estética;

- Melhor cicatrização óssea;

- Melhor modulação da anatomia dos tecidos moles.

\section{DESVANTAGENS DA CARGA IMEDIATA}

A razão risco/benefício deve ser determinada para cada paciente de forma a verificar se a carga imediata é uma alternativa vantajosa.

É um procedimento clinicamente exigente que requer conhecimentos adequados da parte do clínico e colaboração por parte do paciente uma vez que requer mais tempo de consulta de forma a fazer o procedimento cirúrgico e protético num tempo. Para além disso é um protocolo com indicações e critérios de inclusão eexclusão muito restritos, que devem ser respeitados, não podendo ser um procedimento efetuado em todos os pacientes, o que o torna restrito ${ }^{43}$.

\section{RESULTADOS}

São considerados critérios para a utilização de implantes com carga imediata:qualidade do tecido ósseo; propriedades macroscópicas dos implantes (design das roscas); propriedades microscópicas (superfície de revestimento); comprimento mínimo de implante de $10 \mathrm{~mm}$; estabilidade primária absoluta; distribuição dosimplantes, esplintagem primária de implantes que impeçam macromovimentos e uso cauteloso de prótese tipo cantilevers $45,46,47$.

As próteses totais fixas implantossuportas são as preferidas pelos pacientes por proporcionarem conforto e maior eficiência mastigatória, além de menos manutenção e reparo e, favorecendo o aspecto psicológico, uma vez que elimina o caráter removível das overdentures. Todavia, estas próteses agregam maiores custoe uma técnica de confecção mais elaborada, desde o planejamento cirúrgico-protético aos cuidados de controle de biofilme bacteriano ${ }^{48}$.

A carga imediata deve ser colocada em regiões com boa estabilidade imediata,senão poderá haver micromovimentações produzindo formação de tecido fibroso entre o osso e o implante, ocasionando na perda do mesmo ${ }^{49}$. 
A vantagem é que a técnica eliminou o longo período de cicatrização após a colocação do implante e o transtorno dos reembasamentos, nas próteses totais convencionais ${ }^{16}$.

Uma recomendação importante para favorecer a osseointegração durante a reabilitação protética de carga imediata é no possível evitar a realização dos extremoslivres (cantilevers) ou minimizar os extremos, desta forma se diminuir complicações como a fratura da barra ou o afrouxamento dos parafusos agregando ao desenho umaestrutura rígida soldada aos pilares protéticos ${ }^{48}$.

A evolução das técnicas cirúrgicas, o aperfeiçoamento dos métodos de diagnóstico, a melhoria na qualidade do implante em desenho e superfície, bem comoo melhor conhecimento da biologia dos tecidos envolvidos vem dando suporte a estudos com protocolo de único estágio cirúrgico e a colocação de próteses sobre implantes recém instalados ${ }^{50}$.

A rugosidade da superfície do implante aumenta a estabilidade primária uma vez que apresenta maior área de superfície e permite uma ligação mecânica firme aos tecidos circundantes ${ }^{42}$.

Alguns fatores como a biocompatibilidade, o desenho do implante, as condições da superfície do implante, o estado do hospedeiro, a técnica cirúrgica e o controle das cargas após a instalação são determinantes para o sucesso da osseointegração ${ }^{51}$.

Estabilidade primária é a ausência de mobilidade do implante no leito implantarapós ter sido completamente inserido. A base fisiológica em que assenta o conceito da importância da estabilidade primária é a mesma da imobilização após fratura em ossos longos. Não deve haver qualquer movimento entre as suas terminações, de forma a que haja cicatrização da fractura 27.

Acredita que os critérios para a utilização dos implantes em carga imediata são:

- Qualidade do tecido ósseo;

- Macrorrenteções (design da rosca);

- Microrretenções (superfície do implante);

- $\quad$ Estabilização bicortical;

- Distribuição adequada dos implantes;

- Uso cuidadoso de cantilevers; 


\begin{abstract}
Ajuste oclusal adequado enfatizando cargas axiais e minimizandoforças horizontais;

- Utilização de placas noturnas para minimizar possíveis hábitosparafuncionais;

- Próteses provisórias com infra-estrutura rígida para prevenir deflexões ${ }^{1}$.
\end{abstract}

\title{
DISCUSSÃO
}

Segundo Crespi et $a l^{9}$ as vantagens da carga imediata são função e estética imediatas a não necessidade utilizar uma prótese provisória removível não precisa desegunda cirurgia.

Esposito et $\mathrm{al}^{10} \mathrm{em}$ sua última revisão publicada sobre protocolos de carga concluíram que é possível carregar com sucesso os implantes de forma imediata ou precoce em pacientes selecionados, mas as tendências indicam que os implantes decarga imediata fracassam com mais freqüência que os implantes de carga convencional, ainda que com menos freqüência que os implantes de carga precoce.

O fator determinante na definição de carga imediata é a estabilidade inicial. Para Schnitman e colaboradores os implantes com ausência de mobilidade medida pelo perioteste e com boa distribuição no arco, podem ser submetidos à carga imediata. Também Cooper e seus colaboradores, consideram a estabilidade não comoum valor numérico, mas sim a ausência de mobilidade e resistência clínica à rotação.Já para Horiuchi e colaboradores, a estabilidade medida pelo torquímetro, maior ou igual a $40 \mathrm{~N}$, indica a realização de carga ${ }^{11,6,53}$.

Carga imediata é um protocolo bem documentado e previsível em mandíbulas edêntulas ${ }^{34,52}$.

Maxilas totalmente desdentadas têm uma quantidade menor de estudos publicados sobre carga imediata, quando comparados à mandíbula ${ }^{33}$, sendo que vários autores referem não existir dados suficientes que suportem a carga imediata com overdenture, considerando esse procedimento como experimental ${ }^{34,35,36}$.

A pouca literatura existente sugere taxas de sucesso limitadas para a carga imediata em maxilas edêntulas ${ }^{37}$. Concluíram que a maxila edêntula era um local apropriado para carga imediata, com reabilitações fixas totais do arco sobre, no mínimo, 6 implantes ${ }^{2}$. Sugeriu no mínimo 8 implantes ferulizados para reabilitações fixas de carga imediata na maxila ${ }^{38}$. 
O protocolo de carga imediata possui índice de sucesso clínico maior na mandíbula comparado a maxila. Os autores sugerem uma maior quantidade de implantes na maxila, porém se depara com a limitação e qualidade óssea nessa região.

O procedimento em mandíbulas edêntulas tem altas taxas de sucesso, podendo ser recomendado e praticado, o que já não ocorre com tanta frenquência emmaxilas, devido à sua estrutura óssea.

\section{CONCLUSÃO}

Verificou-se que o emprego de carga imediata sobre implantes osseointegráveis constitui uma forma de tratamento com excelentes resultados, desde que se seguem os requisitos necessários para instalação da mesma. Consecutivamente, que sejam planificados e executados os múltiplos fatores indispensáveis para obter o êxito esperado com esse tipo de tratamento. Diante do exposto, o implante é necessário e o protocolo com carga imediata desenvolve a autoestima do paciente após o processo cirúrgico.

A carga imediata é a instalação de implantes dentários seguido de reabilitaçãoprotética logo após a instalação dos implantes ou até 24 horas à 2 semanas após a cirurgia.

O prognóstico de carga mediata em mandíbula é bom, com altos índices de sucesso. Porém o protocolo de carga imediata em maxila é limitado devido a estruturaóssea disponível nessa região.

A técnica traz vários benefícios, visto que no mesmo dia da cirurgia implantar, pode-se instalar a prótese sobre implante. Evita a reabertura do tecido gengival para a instalação de cicatrizadores. O psicológico e autoestima são fatores imensuráveis para o paciente.

\section{REFERÊNCIAS}

1. Salama H, Rose LF, Salama M, Betts MJ. Immediate loading of bilaterally splinted titanium roat-form implants in fixed prosthodontics - a techinique reexamined: two cases reports. Int J Periodont Rest Dent, v. 15, n. 4, p. 345-361, 1995.

2. Dedigi M. et al. (2005). Immediate functional loading of edentulous maxila: a 5- year retrospective study of 388 titanium implants. J Periodontol, 76 (6), pp. 1016- 1024.

3. Buser D. et al. (1988). The new concept of ITI hollow screw implant. Part 2. Clinicalaspects, 
indications and early clinical results. Int J Oral Maxillofac Implants, 3, pp. 173-181.

4. Chiapasco M. et al. (1997). Implant-retained mandibular overdentures with immediate loading. A retrospective multicenter study on 226 consecutive casaes. ClinOral Implants Res, 8, pp. 48-57.

5. Gatti C, Haefglinger W e Chiapasco M (2000). Implant- retained mandibularoverdentures with immediate loading: a prospective study of ITI implants. Int J Oral Maxillofac Implants, 15, pp. 383-388.

6. Horiuchi K. et al. (2000). Immediate loading of Bränemark system implants following placement in edentulous patients: a clinical report. Int J Oral Maxillofac Implants, 15 (6), pp. 824-830.

7. Ioannidou E e Aikaterini D. (2005). Does loading time affect implant survival? A meta-analysis of 1,266 implants. J Periodontol, 76 (8), pp. 1252-1258.

8. Atieh MA, Aisabeeha NH e Payne AG. (2012). Can resonance frequency analysis predict failure risk of immediately loaded implants? Int J Prosthodont, 25(4), pp. 326- 39.

9. Mah J, Bergstrand F. Temporary anchorage devices: a status report. J Clin Orthod.,2005 mar;9(3): 132-36.

10. Crespi R. Immediate occlusal loading of implants placed in fresh sockets after tooth extraction. Int J Oral Maxillofac Implants 2007, 22:955-62.

11. Schnitman PA, Whorle PS, Rubenstein JE et al. Ten years results for Brånemark implants immediately loaded with fixed prosthesis at implant placement. Int. J. Oral Maxillofac. Implants, v. 12, p. 495-503, 1997.

12. Tarnow DP, Emtiaz S, Classi A. Immediate loading of threaded implants at stage 1 surgery in edentulous arches: tem consecutive case reports with 1-to 5-year data. Int. J. Oral Maxillofac. Implants, v. 12, p. 319-324, 1997.

13. Guerrieri GL, Miguel RC. Implantologia oral. Rio de Janeiro: Ed. Guanabara/Koogan, 1982; 21-46.

14. Costa RR, Vaz MAK. Implante imediato com carga imediata em unitários anteriores relato de caso clínico. Revista Bras Cirur. Prótese e Implant. v. 7, n. 28, p. 69-72, out./dez. 2000. In: MULLER, A. et al. Implante unitário submetido à cargaimediata. RGO, v. 52, n. 1, p. 27-41, jan./fev./mar. 2004.

15. Esposito M, Hirsch JM, Lekholm U, Thomsen P. Biological factors contributing tofailures 
of osseointegrated oral implants. (I). Success criteria and epidemiology. European Journal of Oral Sciences, v. 106, n. 1, p. 527-551, 1998.

16. Ferraz RG, Menezes JCPX, Anuar A. Protocolo de carga imediata sobre implantes em mandíbula edentada - Relato de caso clínico. Rev. ABO Nac. Suplemento, v. 18 n. 1 - fevereiro/março 2010

17. Bechelli $\mathrm{AH}$. Carga imediata em implantodontia oral: protocolos diagnósticocirúrgicos protéticos casos clínicos. Livraria Santos Editora- São Paulo. 2005.

18. Cochran DL et al. (1998). Bone response to unloaded and loaded and loaded titanium implants with a sandblasted and acid-etched surface: A histometric study inthe canine mandible. J Biomed Mater Res, 40 (1), pp. 1-11.

19. Mcclarence, Elaine. Close to the Edge - Branemark and the Developmente of Osseointegration. Berlim: Quintessence Books, 2003.

20. Lee JH, Frias V, Lee KW, Wright RF. Effect of implant size and shape on implantsuccess rates: a literature review. J Prosthet Dent 2005; 94:377-81.

21. Meredith N. Assessment of Implant Stability as a Prognostic Determinant. The International Journal of Prosthodontics, v. 11, n. 5, p. 491-502, 1998.

22. Barros GPC, Neto SBR. Carga imediata em implantes unitários: revisão de literatura. Arqu bras odontol. v. 6, n. 3, p. 163-9, 2010.

23. De Brandão ML et al. Superfície dos implantes osseintegrados $\mathrm{x}$ respostabiológica. Revista ImplantNews. v.7, n.1, p.95-101, 2010

24. Albrektsson T. A multicenter report on osseointegrated oral implants. J Prosth Dentistry 1988;60(1):75-84.

25. Van Steenberghe D, Lekholm U, Bolender C, Folmer T, Henry P, Herrmann I et al. The applicability of osseointegrated oral implants in the rehabilitation of partial edentulism: a prospective multicenter study on 558 fixtures. Int J Oral Maxillofac Implants 1990;5:272-81.

26. Misch C. E. et alii. (2004b). Rationale for the application of immediate load in implant dentistry: Part II. Implant Dent, 13 (4), pp. 310-321.

27. Javed F, Romanos GE (2010). The role of primary stability for successful immediate loading of dental implants. A literature review. Journal of Dentistry, 38, pp.612-620.

28. Borges PB. Tratamento da superfície de implantes. 2013. $27 \mathrm{f}$. Trabalho de conclusão de curso (especialização em Prótese dentária) - Universidade Federal de Minas Gerais, 2013.

29. Misch CE et al. (2004a). Workshop guidelines on immediate loading in implant dentistry. Journal Oral Impl, 30 (5), pp. 283-288. 
30. Misch CE et al. (2004b). for the application of immediat load in implant dentistry:Part II. Implant Dent, 1 (4), pp.310-321.

31. Degidi M et al. (2007). Do longer implants improve clinical outcome in immediate loading? Int J Oral Maxillofac Surg, 36, pp. 1172-1176.

32. Vidyasagar L. e Apse, P. (2004). Dental implant design and biological effects onboneimplant interface. Stomatologija, Baltic Dental and Maxilofacial Journal, 6 (2), pp. 51-54.

33. Ostman P. (2000). Immediate loading of dental implants. Clinical documentationand presentation of a treatment concept. Periodontology, 47, pp. 90-112.

34. Cochra DL, Morton D. e Weber HP. (2004). Consensus treatments and recommended clinical procedures regarding loading protocols for endosseous dental implants. Int J oral Maxillofac Implants, 19, pp. 109-113.

35. Galluci GO, Morton D. e Weber HP. (2009). Loading protocols for dental implantsin edentulous patients. Int J Ora Maxillofac Impantsl, 24, pp. 132-146.

36. Weber HP et al. (2009). Consensus statements and recommended clinical procedures regarding loading protocols. Int J Oral and Maxilofac Implants, 24 (suppl), pp. 180-183.

37. Attard NJ e Zarb GA. (2005). Immediate and early implant loading protocols: A literature review of clinical studies. The Journal of Prosthetic Dentistry, 94(3), pp. 242-258.

38. Misch CE (2008a). Carga Imediata na Implantologia: considerações e tratamento.In: Misch CE (Ed), Implantes Dentais Contemporâneos, Editora Elsevier, 3ạ edição, pp. 799-836.

39. Cochran DL (2006). The evidence for immediate loading of implants. J evid BaseDent Practice, 6, pp. 155-163.

40. Kher U e Patil, S. (2011). Immediate loading of implants in the mandible. Int J Orallmpl Clinical Res, 2 (1), pp. 49-53.

41. Corso $\mathrm{M}$ et al. (2012). Clinical and radiografic evaluation of early loaded freestanding dental implants with various coatings in beagle dogs. J Prosthet Dent, 113 (3), pp. 35-40.

42. Javed F. e Romanos GE. (2010). The role of primary stability for successful immediate loading of dental implants. A literature review. Journal of Dentistry, 38, pp.612-620.

43. Ho CCK (2005). Immediate function with dental implants. Dent Pract, pp 156-166. [Emlinha]Disponívelem<http://www.idimplants.com.au/pdf/Implant\%20lmmediate\%20 loading\%20article.pd f> [Consultado em 12/09/2012]. 
44. Degidi M. et al. (2008). Immediately loded titanium implant with a tissuestabilizing/maintaining design ('beyond platform switch') retrieved from man after 4 weeks: a histological and histomorphometrical evaluation. A case report. Clin Oral Impl Res, 19, pp. 276-282.

45. André LFM, Amaral JMBL, Massote A et al. Carga imediata através de coroas telescópicas. Rev Bras Implantod Prótese Implant; v.10, n.37, p. 34-39. 2003.

46. De Leo C, Geremia T, Lacroix C. et al. Carga imediata em implantes osseointegrados inclinados: aumentando a superfície de ancoragem - Relato de doiscasos. Rev Odont Cienc; v.17, n.38, p.331-338.2002.

47. Marcantonio EJ, Grisi DC. Aplicação de Carga Imediata em Implantes Dentais. Revista Brasileira de Cirurgia e Implantodontia, v. 9, n. 34, p. 111-116. abr/jun. 2002.

48. Rocha SS, Souza DR, Fernandes JMA, Garcia RR, Zavanelli RA. Próteses TotaisFixas Tipo Protocolo Bimaxilares. Relato de Caso. Rev Odontol Bras. Central v.21, n.60, 2003.

49. Fusaro BF, Oliveira RG, Sotto Maior BS, Sotto Maior HF, Vieira DF. Prótese total inferior implantosuportada com carga imediata. Estação Científica. 2005. Disponível em: http://portal.estacio.br/media/3344103/5- protese-total-inferior-implanto- suportadacargaimediata.pdf. Acesso: 05/11/2021.

50. Barros GC. et al. Carga imediata em implantes unitários: revisão de literatura Arqubras odontol 2010;6(3):163-9.

51. Albrektsson T, Brånemark P.-I, Hansson H.A. Lindström J. Osseointegrated titanium implants. Requirements for ensuring a long-lasting, direct bone-to-implant Anchorage inman. Acta Orthopaedica Scandinavica 1981; 52:155-70

52. Testori T. et al. Systematic Review of Survival Rates for Immediately Loaded Dental Implants. Internat Jour of Periodont \& Restorat Dent, v. 3, n. 26, p. 3-17, 2006. 
53. Cooper LF, Rahman A, Moriarty J, Chaffee N, Sacco D. Immediate mandibular reabilitation with endosseous implants: simultaneous extraction, implant placement and loading. Int J Oral Maxillofac Implants, v. 17, n.4, p. 517-525, 2002. 
